\title{
Systems genetics identifies a macrophage cholesterol network associated with physiological wound healing
}

\author{
Marta Bagnati, ${ }^{1}$ Aida Moreno-Moral, ${ }^{2}$ Jeong-Hun Ko, ${ }^{1}$ Jérôme Nicod, ${ }^{3}$ Nathan Harmston, ${ }^{2}$ \\ Martha Imprialou, ${ }^{1}$ Laurence Game, ${ }^{4}$ Jesus Gil, ${ }^{5}$ Enrico Petretto, ${ }^{2,6}$ and Jacques Behmoaras ${ }^{1}$ \\ ${ }^{1}$ Centre for Inflammatory Disease, Imperial College London, Hammersmith Hospital, London, United Kingdom (UK). \\ ${ }^{2}$ Duke-NUS Medical School, Singapore, Singapore. ${ }^{3}$ Wellcome Centre for Human Genetics, University of Oxford, Oxford, UK. \\ ${ }^{4}$ Genomics Laboratory, Medical Research Council (MRC) London Institute of Medical Sciences, Imperial College London, \\ Hammersmith Hospital, London, UK. ${ }^{5}$ Cell Proliferation Group, MRC London Institute of Medical Sciences (LMS), London, \\ UK. ${ }^{6}$ MRC London Institute of Medical Sciences, Faculty of Medicine, Imperial College London, London, UK.
}

Among other cells, macrophages regulate the inflammatory and reparative phases during wound healing but genetic determinants and detailed molecular pathways that modulate these processes are not fully elucidated. Here, we took advantage of normal variation in wound healing in 1,378 genetically outbred mice, and carried out macrophage RNA-sequencing profiling of mice with extreme wound healing phenotypes (i.e., slow and fast healers, $n=146$ in total). The resulting macrophage coexpression networks were genetically mapped and led to the identification of a unique module under strong trans-acting genetic control by the Runx2 locus. This macrophagemediated healing network was specifically enriched for cholesterol and fatty acid biosynthetic processes. Pharmacological blockage of fatty acid synthesis with cerulenin resulted in delayed wound healing in vivo, and increased macrophage infiltration in the wounded skin, suggesting the persistence of an unresolved inflammation. We show how naturally occurring sequence variation controls transcriptional networks in macrophages, which in turn regulate specific metabolic pathways that could be targeted in wound healing.

License: This work is licensed under the Creative Commons Attribution 4.0 International License. To view a copy of this license, visit http:// creativecommons.org/licenses/ by/4.0/.

Authorship note: MB and AMM contributed equally to this work.

Conflict of interest: The authors have declared that no conflict of interest exists.

License: Copyright 2019, American Society for Clinical Investigation.

Submitted: October 22, 2018 Accepted: December 18, 2018 Published: January 24, 2019

\section{Reference information:} JCI Insight. 2019;4(2):e125736 https://doi.org/10.1172/jci. insight.125736.

\section{Introduction}

Wound healing is the repair of damaged and injured skin. It is a complex and highly dynamic process consisting of overlapping phases that include inflammation, tissue formation and remodeling, which can lead to the production of a nonfunctioning mass of fibrotic tissue known as the scar (1). Impaired wound healing is a critical socioeconomic problem in the Western world (2), as chronic nonhealing wounds represent a clinical burden associated with significant healthcare costs (3).

Among other cells, macrophages are pivotal effector cells during wound healing. They dynamically change their activity throughout the healing process as their immune/phagocytic properties gradually convert into a more reparative and immunomodulatory phenotype $(4,5)$. Macrophages are present during all stages of the repair process and their depletion with anti-macrophage serum results in impaired wound healing (6). In addition, conditional depletion of macrophages during the different phases of wound healing has further confirmed their context-dependent plasticity (7). Different macrophage polarization stages correspond to different phases of tissue repair: proinflammatory macrophages infiltrating during the initial inflammatory phase are later antagonized by immunomodulatory macrophages that promote tissue repair and fibrosis $(5,8)$.

Animal model systems can provide a useful tool to map pathways and genes underlying (patho)physiological traits $(9,10)$. For instance, several inbred mouse strains differ significantly in their rate of wound healing (11) and some of these strains were used in genetic linkage studies aiming to map quantitative trait loci $(12,13)$ controlling physiological wound healing. A significant advance in gene mapping strategies came from genome-wide association studies (GWAS) carried out in genetically outbred mice, which dramatically improved mapping resolution over traditional quantitative trait locus (QTL) studies (14). Outbred mice, which are bred to maintain maximum heterozygosity (15), offer the advantage of gene-level mapping 
resolution due to relatively restrictive haplotype blocks and a large catalog of variants that can be tested for association (16).

Previously, a GWAS of multiple complex traits using a commercially available outbred mouse population achieved single-gene resolution mapping for multiple traits, including behavioral, physiological, and tissue phenotypes (14). The outbred mouse population offers a lower significance threshold and a smaller sample size in comparison with human GWAS (14). Quantitative variation in wound healing was one of the 92 phenotypes measured in almost 2,000 outbred mice, and importantly, this trait was associated with a single gene (Bmp2) following genetic mapping using low-coverage sequencing (14). Bmp2, and more generally Bmp signaling, was previously found to be involved in wound healing $(17,18)$ but the significance of this result in terms of the effector cell function in wound healing remained to be determined. Genotype-phenotype relationships, often first identified by GWAS, could be integrated with systems genetics approaches aiming to reveal the cellular and molecular pathways underlying complex traits (19). Geneand/or network-level strategies in the relevant effector cells could complement GWAS in functionally annotating genes $(20,21)$. We therefore hypothesized that naturally occurring germline sequence variation could affect macrophage function associated with physiological wound healing.

To test this hypothesis, we used the ear punch model to study the rate of wound healing in 1,378 outbred mice. We integrated phenotypic and genotypic analyses in this population (i.e., ear area closure after healing and genome-wide single-nucleotide polymorphism [SNP] genotyping) with whole-genome mRNA sequencing (RNA-seq) in primary macrophages from mice with extreme wound healing phenotypes (i.e., fast and slow healers from normal distribution of the rate of wound healing). This led to the identification of a gene network enriched for fatty acid and cholesterol biosynthesis that (a) associates with the rate of wound healing and (b) is under strong genetic control in trans by the transcription factor Runx2 located on mouse chromosome 17. We showed that inhibiting the DNA binding activity of Runx2 affects the expression of the genes in this network. We then targeted Fasn, which catalyzes the synthesis of long-chain fatty acids and also shows a regulatory role in cholesterol synthesis (22). In vivo pharmacological blockage of Fasn results in delayed wound healing and increased macrophage recruitment in the skin wound, suggesting the persistence of an unresolved inflammation. These results suggest the importance of fatty acid/ cholesterol biosynthesis in the healing process.

\section{Results}

The rate of wound healing is normally distributed in a large outbred mouse population. Outbred mice are large, genetically heterogeneous colonies that commercial mouse breeders maintain. Typically, these colonies present the necessary genetic structure and variation to allow high resolution mapping of complex traits, which can be exploited for gene identification by GWAS. A 2-mm ear punch was performed in 16-week-old CFW outbred mice and wound healing monitored after 5 weeks (ear area after healing) in 1,378 outbred animals as part of a larger study combining phenotyping of multiple traits and genotyping by low-coverage sequencing (14). We reasoned that the major molecular determinants underlying this process could be more easily captured by selective RNA-seq of the macrophages of fast and slow healers $(23,24)$. One hundred forty-six mice were selected for this purpose at the extreme of the ear area distribution (62 fast healers and 84 slow healers) and their bone marrow-derived macrophages (BMDMs) were analyzed by RNA-seq (Supplemental Table 1; supplemental material available online with this article; https://doi.org/10.1172/jci. insight.125736DS1). The available resources for these 146 mice were (a) quantitative differences in wound healing indicated as ear area, (b) BMDM mRNA expression by RNA-seq, and (c) genome-wide SNP genotyping imputed from low-coverage sequencing (Figure 1).

A macrophage-mediated healing network maps strongly to the Runx2/Supt3h locus. Instead of mapping the genetic control of single gene expression, we used a gene coexpression module/network identification and mapping strategy, which we have previously applied to identify trans regulators of networks in complex diseases (25-27). First, we inferred macrophage gene coexpression modules from the transcriptome of fast and slow healers. This analysis identified 40 coexpression modules, each containing 30 to 1,151 genes, listed in Supplemental Table 2.

To identify key regulators of the transcriptional programs captured by the coexpression modules inferred in the fast and slow healers, we carried out network QTL mapping (28). This analysis resulted in a unique module that was strongly associated (Bayes factor $[\mathrm{BF}]=211,096.6$ ) with a SNP located on mouse chromosome 17 (Figure 2A). The strong association of this module (designated as "macrophage-mediated healing 


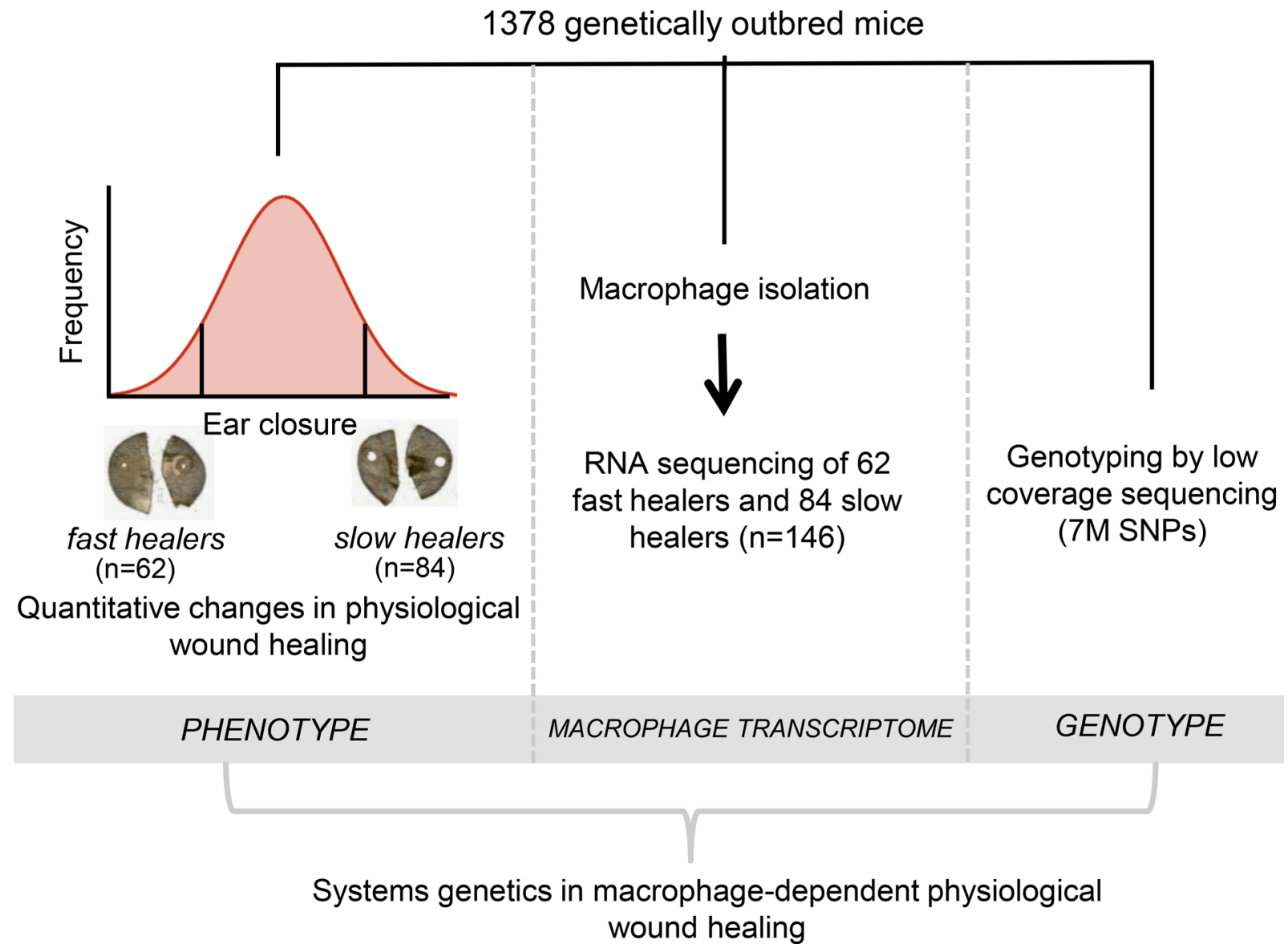

Figure 1. A systems genetics approach for deciphering macrophage-dependent wound healing. Study design showing 1,378 outbred mice subjected to a 2-mm ear punch and wound healing measured after 5 weeks. One hundred forty-six mice were selected at the extreme of the ear area distribution (62 fast healers and 84 slow healers) and their macrophages analyzed by RNA sequencing (RNA-seq). The available resources for these 146 mice are (i) quantitative differences in wound healing indicated as ear area following punch, (ii) RNA-seq in bone marrow-derived macrophages (BMDMs), and (iii) genomewide SNP genotyping by low-coverage sequencing.

network" [MMHN]) is also illustrated by its 195-fold increase in BF when compared with the second strongest associated module $(\mathrm{BF}=1,084.5)$. Rather than adopting a nearest gene approach to investigate the putative target gene for the regulatory SNP, we interrogated publicly available high-resolution chromatin interaction data (29). This revealed that the regulatory SNP (chr17_45131552) is located within a highly conserved and well-characterized topologically associated domain (TAD), containing both Runx2 and Supt3h genes (Figure 2B). TADs have been proposed to represent the regulatory domains of the genes located within them (30), primarily by delimiting the scope of enhancer-promoter interactions $(31,32)$. The TAD that contains the SNP associated with the MMHN is highly conserved across tissues and species (33-35) and found in human macrophage Hi-C data (36) (Supplemental Figure 1). The analysis of additional candidate trans-acting regulators upstream the macrophage gene coexpression networks associated with wound healing is reported in Supplemental Figure 2.

The MMHN correlates with wound healing and is under Runx2 trans regulation. Functional enrichment analysis of the MMHN showed strong and specific enrichment for cholesterol and fatty acid biosynthetic processes (Figure 3A). Closer inspection of the network genes showed the presence of Hmgcr, the rate-limiting enzyme in cholesterol synthesis, together with Lss and Dhcr7, which are positioned downstream of Hmgcr. Furthermore, Fasn, which catalyzes the synthesis of palmitate, has been recently linked to cholesterol biosynthesis in macrophages (22).

We investigated Runx2 transcription factor binding sites (TFBS) in the promoter of MMHN genes and identified 70 genes with a predicted Runx2 TFBS (Figure 3A), further suggesting a trans-acting genetic regulation of this network by Runx 2 . 


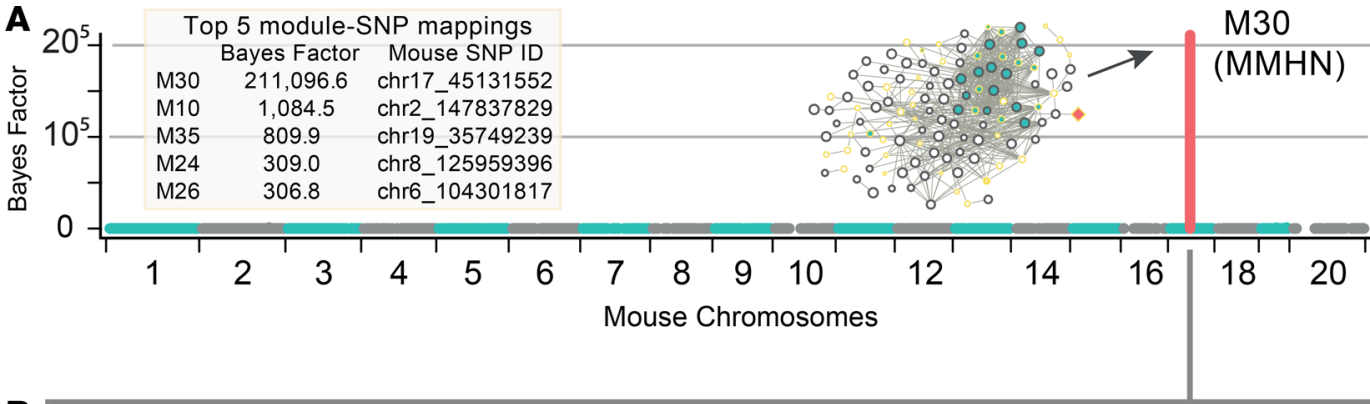

B

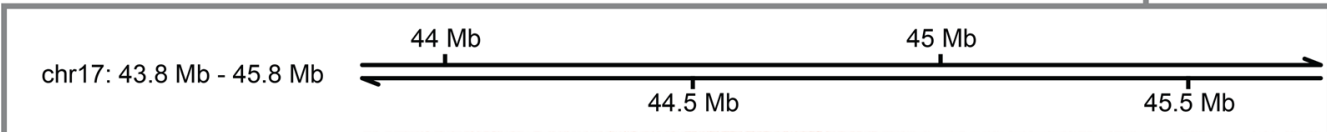

Hi-C matrix

(Mouse Embryonic

Stem Cells)

\section{Topologically associating} domains (TADs)

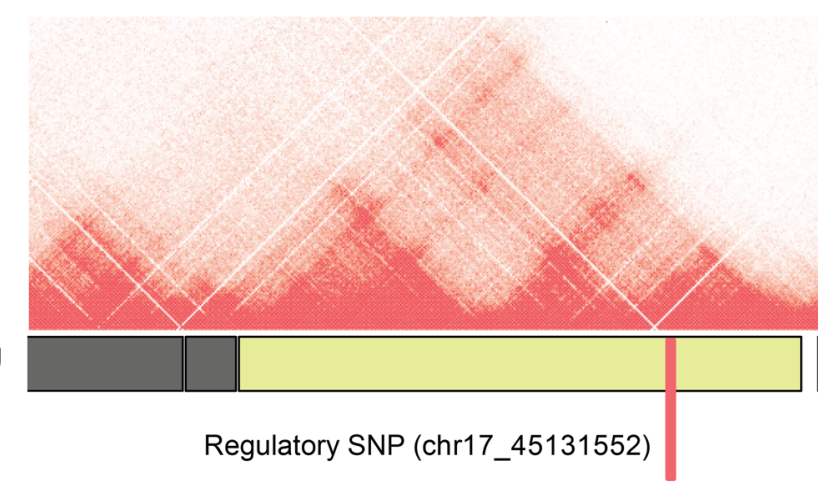

$5.5 \mathrm{Mb}$

in chromosome 17
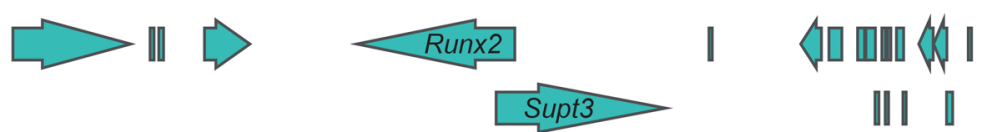

III |

Figure 2. Strong association of a macrophage-mediated healing network with the Runx2/Supt3h locus. (A) Genomewide mapping results from multivariate Bayesian mapping of 40 macrophage coexpression modules in the outbred mouse population showing fast and slow healing $(n=146)$. The coexpression module with the highest association (Bayes factor [BF] of 211,096) is M30 (also designated as macrophage-mediated healing network, MMHN) through SNP (chr17_45131552). The results for the top 5 modules with strongest genetic association are presented in a table (left). (B) M30 is associated with chr17_45131552 on mouse chromosome 17, which is located within a well-conserved and tissue invariant (Supplemental Figure 1) topologically associated domain (TAD) containing Runx2 and Supt3h in mouse embryonic stem cells. As TADs can represent the regulatory domain of the genes they contain, the location of chr17_45131552 within this TAD implicates it in the transcriptional regulation of either Runx2 or Supt3h. See supplemental methods for more details.

We set out to inspect the relationship between the transcriptional regulation of the MMHN by Runx2 and wound healing using a 2-step approach. We first correlated the variance-stabilized transformed (VST) gene counts of all transcripts in the network with Runx 2 expression. We then correlated the VST gene counts of all the transcripts in the network with the rate of wound healing (see supplemental methods). These analyses showed that the transcripts in the network that positively correlate with Runx2 expression also positively correlate with healing, and the same is true for those correlating negatively ( $\rho=0.74, P=1.04^{-31}$ ) (Figure 3B). Taken together, these results showed that the genetically controlled MMHN is enriched for genes implicated in cholesterol and fatty acid biosynthetic processes, whose expression correlates with Runx 2 mRNA expression and the rate of wound healing.

In order to validate Runx2 regulation of MMHN genes in vitro, we cultured BMDMs and incubated them for 48 hours with $20 \mu \mathrm{M}$ CADD522, a drug previously shown to selectively block Runx2 DNA binding activity (37). Expression of genes positively and negatively correlated with Runx2 was then assessed by quantitative reverse-transcription PCR (qRT-PCR). In line with the correlation identified in silico, we found significant downregulation of transcripts showing a positive correlation with Runx2 (Med23, Med17, Rsrp1, P4ha1, Rbm5, Nfkbiz, Kdm1a, and Jmjd4) (Figure 3C), and significant upregulation of the genes having negative correlation with Runx2 (Fasn, Acly, Hmgcr, Prkch, Fdps, Dhcr7, Mvk, Lss, and Poc1b) (Figure 3D). These results confirm the role of Runx2 as a trans-genetic regulator of MMHN genes in murine BMDMs. 
A Functional enrichment of MMHN (-Log ${ }_{10}$ FDR)

$$
\begin{aligned}
& \text { cholesterol biosynthetic process }
\end{aligned}
$$

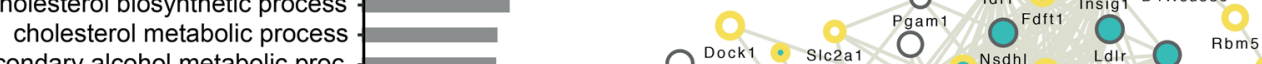

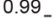

$$
\begin{aligned}
& 0.60
\end{aligned}
$$
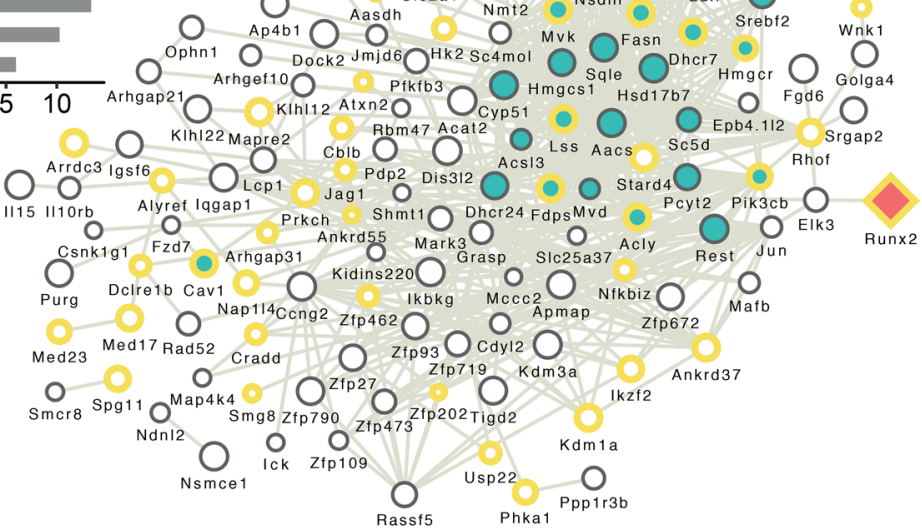

\begin{tabular}{|lll}
\hline 10 & \\
5 & 10
\end{tabular}

B

c Genes positively correlating with Runx2

Correlation of MMHN genes with Runx2 versus correlation of MMHN genes to with healing
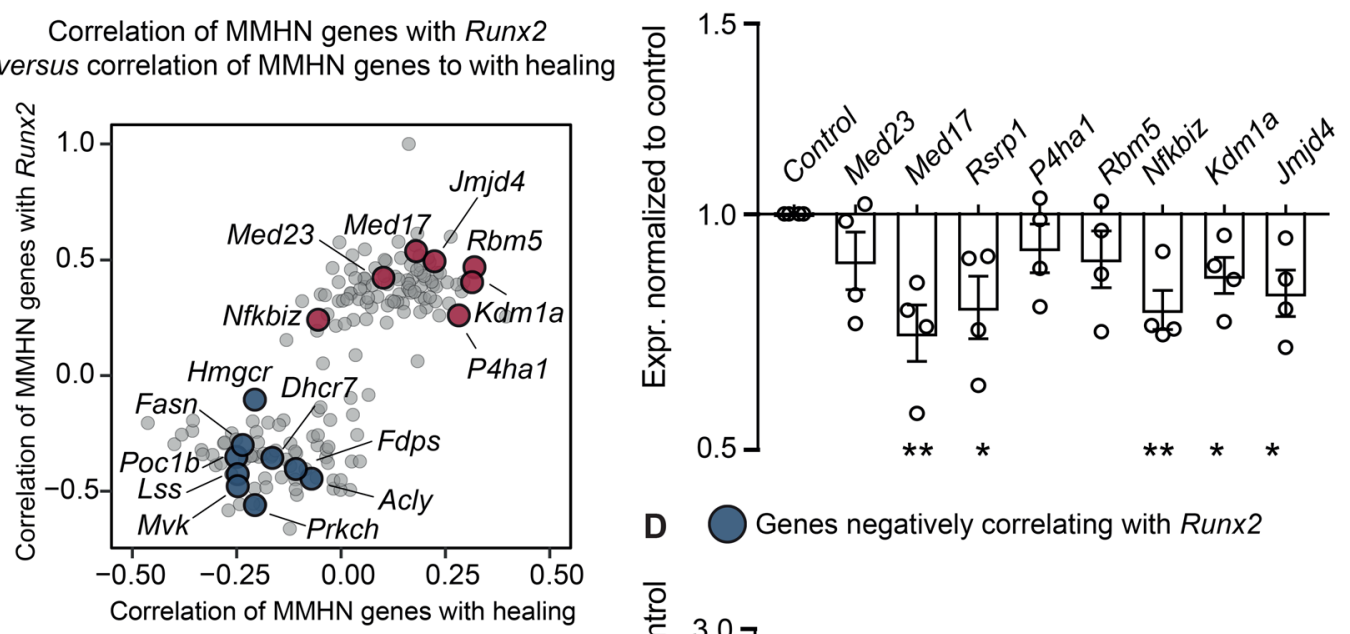

D Genes negatively correlating with Runx2

Fatty acid and cholesterol pathway genes in MMHN validated by $\mathrm{qPCR}$

Positive correlation with Runx2

Negative correlation with Runx2

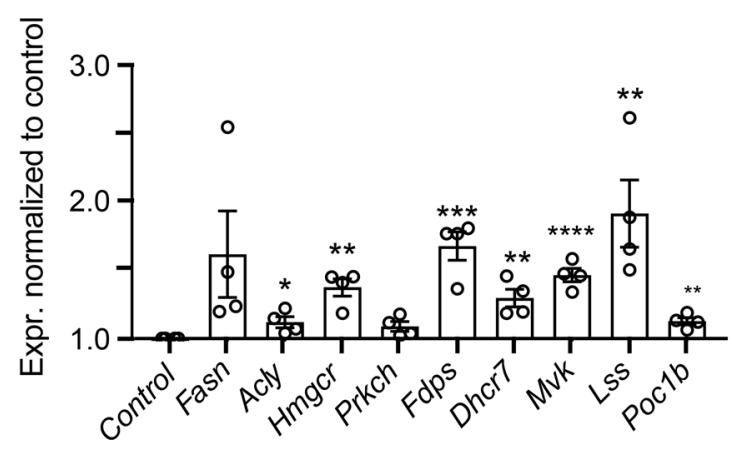

Figure 3. The macrophage-mediated healing network is enriched for cholesterol/lipid biosynthesis and is partly controlled by Runx2 activity. (A) Overview of coexpression module M30 (MMHN). Top left: Gene Ontology (GO) functional enrichment of genes in MMHN (177 genes). Network graph with the genes (nodes) in the MMHN that have any known protein-protein, coexpression, and database interactions (edges) in the STRING protein database (67). Node size represents the degree of probability of association for each gene to the regulatory SNP chr17_45131552 (computed by Bayesian multivariate mapping). Genes with a predicted $R U N X 2$ transcription factor binding site (TFBS) in their promoter are shown with a yellow border. Genes annotated with the GO functional term "lipid biosynthetic process" are presented in green. Runx2 (trans regulator) is colored in orange. See supplemental methods for additional details. (B) Genes whose expression levels correlate with Runx2 also correlate with the rate of healing. Spearman's correlation of the expression level of each gene in the MMHN with Runx2 expression levels (normalized variance-stabilized counts), against the correlation between the expression levels of each gene in the MMHN and the rate of healing ( $\rho=0.74, P=1.04^{-31}, n=146$ mice). ( $C$ and $\left.\mathbf{D}\right)$ qRT-PCR results of a subset of MMHN genes showing positive and negative correlation with $R$ Runx2, respectively, following Runx2 blockage with CADD522 $(20 \mu \mathrm{M})$ in mouse BMDMs. Data are expressed as mean $\pm \operatorname{SEM}(n=4)$. ${ }^{*} P<0.05$, ${ }^{* *} P<0.01,{ }^{* *} P<0.005$, ${ }^{* * * *} P<0.001$ by 2 -tailed Student's $t$ test.

Pharmacological targeting of Fasn in vivo delays wound healing in rats. In order to assess the effect of the modulation of the MMHN in vivo, we pharmacologically targeted one of its genes, Fasn, using cerulenin, a fatty acid synthase blocker (38). We reasoned that the modulation of a regulator of both fatty acid and cholesterol pathways (22) might accurately reflect MMHN in the healing process. 
A

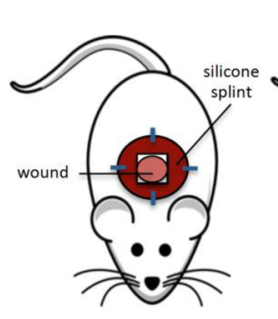

Day 0 Wound healing

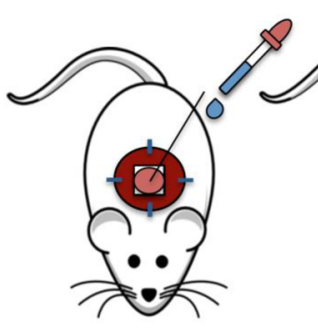

Day 0 Cerulenin/vehicle treatment

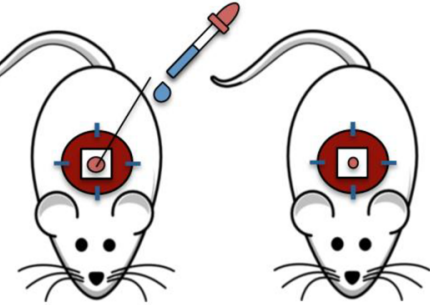

Day $3 / 6$ Cerulenin/vehicle treatment
Day 8 Wound measurement
B

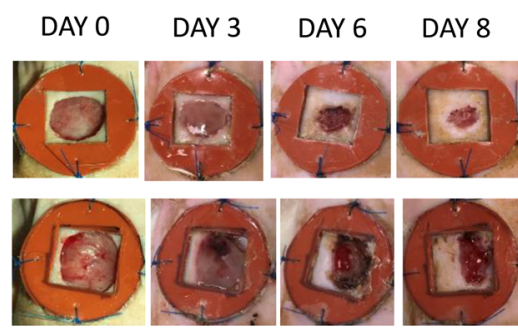

D

control

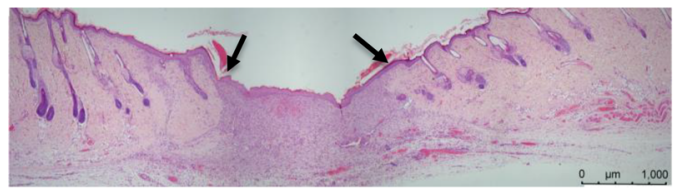

cerulenin

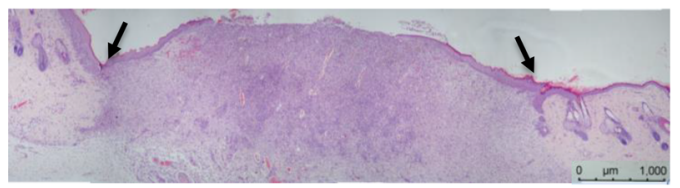

$\mathbf{F}$

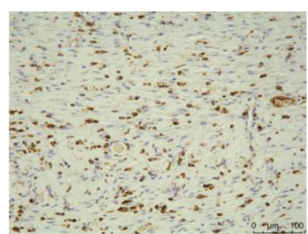

control

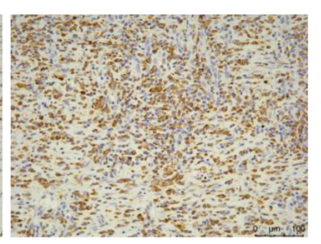

cerulenin

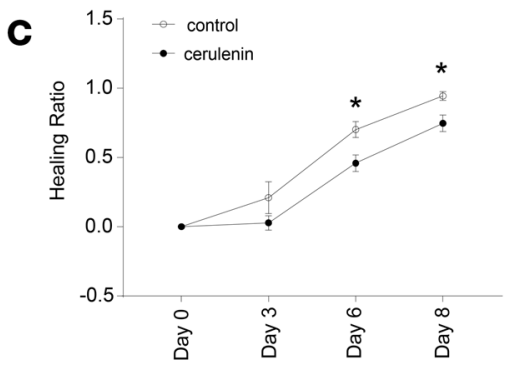

E

control

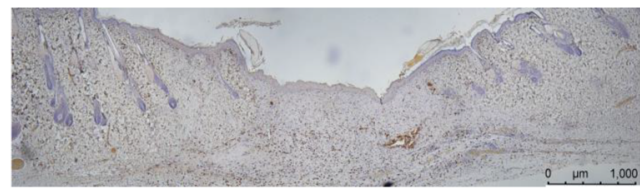

cerulenin

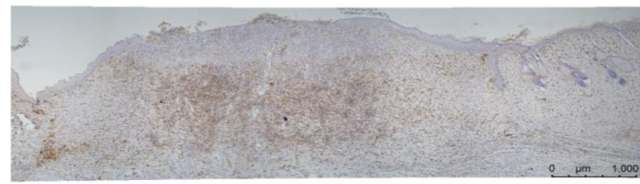

G

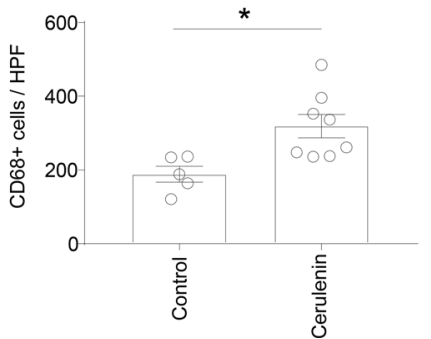

Figure 4. FASN inhibition with cerulenin affects wound healing in the skin. (A) Experimental design. Full-thickness, 10-mm splinted excisional wounds were performed on the dorsal skin of 13-week-old Lewis (LEW) rats. Wounds were treated with $300 \mu \mathrm{g}$ of cerulenin in propylene glycol at 0,3 , and 6 days after the excision or with propylene glycol alone (vehicle). Tissues were collected 8 days following wounding. (B) Representative images of wounds immediately following surgery (day 0 ) and after 3, 6, and 8 days for control and cerulenin-treated rats. (C) Quantification of wound area at days 0, 3, 6, and 8 after wounding. Values are expressed as the healing ratio in comparison to day 0 and are represented as mean $\pm \operatorname{SEM}(n=6$ controls and $n$ $=8$ cerulenin treated). (D) Representative image of $\mathrm{H} \& \mathrm{E}$-stained wounds 8 days after wounding in control and cerulenin-treated rats. Black arrows indicate the edges of granulation tissue. (E) Representative CD68 (rat ED-1) immunohistochemistry within the wound edges in control and cerulenin-treated rats. (F) CD68 staining showing the center of the granulation tissue. (C) Quantification of CD68 ${ }^{+}$cells per high-power field $(\mathrm{HPF}, \times 20)$ in control and cerulenin-treated wounds. Data are expressed as mean \pm SEM ( $n=5$ controls and $n$ $=8$ cerulenin treated. Scale bars: $1,000 \mu \mathrm{m}$ (D and $\mathbf{E})$ and $100 \mu \mathrm{m}$ (F). ${ }^{*} P<0.05$ by 2 -tailed Student's $t$ test.

Full-thickness, 10-mm splinted excisional wounds were inflicted on the dorsal skin of 13-weekold Lewis (LEW) rats. By splinting the wounds, contraction is minimized to favor granulation and reepithelialization, which characterizes wound healing in humans. Skin wounds were treated with 300 $\mu \mathrm{g}$ of cerulenin in propylene glycol at 0,3 , and 6 days after wounding. Histological analysis was performed at day 8 after wounding (Figure $4 \mathrm{~A}$ ). Wounds treated with cerulenin showed a significant delay in healing at both day 6 and day 8 after wounding (Figure 4, B-D) and were associated with increased fatty acid synthesis/cholesterol genes (Supplemental Figure 3). Immunohistochemical analysis of the macrophage marker CD68 showed a significantly increased number of $\mathrm{CD} 68^{+}$cells in the wounds treated with cerulenin (Figure 4, E-G), suggesting the delayed wound healing observed with cerulenin treatment can be accompanied by an unresolved inflammation characterized by persistent macrophage infiltration. Overall, these results suggest that the modulation of the MMHN in vivo can affect the rate of wound healing in rats. 


\section{Discussion}

Physiological wound healing involves highly interactive and dynamic processes including inflammation, angiogenesis, matrix deposition, and cell recruitment, which can only be under polygenic and complex genetic control in genetically outbred populations. Evidence from several studies conducted in murine and porcine models support the idea that physiological wound healing is indeed heritable (39-41). While there is genetic association evidence for the pathological forms of wound healing such as diabetic foot ulcers (42), keloids (43), and chronic venous disease (44), the only GWAS for physiological wound healing was conducted in a commercially available outbred population, which allowed single-gene-resolution mapping and identification of causal variants (14). Here, we performed RNA-seq of macrophages derived from fast and slow healers identified from the same outbred population. We show that a MMHN controlled by Runx2 associates with wound healing through cholesterol/fatty acid biosynthesis. These results complement the GWAS carried out in the same population where Bmp2 was the only gene identified as being associated with wound healing. Runx2 is downstream of Bmp2 in osteoblasts $(45,46)$, which suggests that our systems genetics approach broadened the GWAS results by identifying variants that could affect wound healing through macrophage function.

In our gene network mapping strategy, we used BMDMs as a proxy for wound macrophages in the outbred mice. Besides the methodological advantages (i.e., cell number, control of environmental factors), there is evidence based on the green fluorescent protein-bone marrow chimeric mouse models of wound healing showing that macrophages at the wound healing originate predominantly from the bone marrow (47). In addition, it is possible that the RUNX2 trans-acting genetic regulation of the network is conserved across the myeloid lineage, given previous studies showing the conservation of cis-expression QTLs in monocytes/macrophages in general (48).

The evidence for Runx2 involvement in skin wound healing is scarce (49), despite a large body of work implicating its role in skeletal development (50) and more specifically in osteoblast and terminal chondrocyte differentiation (51). In keeping with this, SNPs located at the Runx2-Supt3h locus have been associated with bone and cartilage phenotypes by GWAS (52), which suggests shared genetic control of physiological wound healing rates and bone and cartilage phenotypes. The role of Runx 2 in immune cells and more particularly in macrophages is not well documented. It has been reported that RUNXs are involved in NOD2 stimulation in human macrophages (53) and that RUNXs mediates egress of plasmacytoid dendritic cells into the circulation (54), controlling their development and migration (55). The exact role of Runx2 in macrophage function remains to be established in the context of macrophage plasticity during the healing process. In addition, the previously established physical proximity between the promoters of Runx2 and Supt3h (34) suggests a coordinated trans regulation of the MMHN. Thus, we cannot rule out a subgroup of genes belonging to the network that are under Supt3h control, especially given its ability to regulate ADAMTS13 activity (56), which has been linked to wound healing (57).

Recent advances in the metabolic control of innate immune function will have a considerable impact on manipulating the rate of wound healing, given the known plasticity of macrophage function depending on context-dependent metabolic pathways (5). The importance of targeting HIF-1 $\alpha$, a hallmark of proinflammatory, glycolytic macrophage activation in wound healing (58) and, more recently, the lactic aciddriven delivery of repair agents such as CXCL12 (59) show how the healing process is tightly dependent on the metabolic status of the wound microenvironment.

The regulation of the macrophage inflammatory response by cholesterol metabolism previously revealed a role of lanosterol (the first sterol intermediate in the cholesterol biosynthetic pathway) in modulating the TLR4 response (60). Similarly, desmosterol, the last intermediate in the Bloch pathway of cholesterol biosynthesis, has been linked to the suppression of inflammatory-response genes in macrophages (61). Furthermore, the oxysterol 25-hydroxycholesterol (25-HC) acts by antagonizing sterol response element-binding protein (SREBP) processing to reduce IL- $1 \beta$ transcription and to broadly repress IL-1-activating inflammasomes (62). Selective deletion of FASN in macrophages protects again inflammation (63), and interestingly, macrophages lacking FASN had reduced cholesterol levels (63), suggesting the regulatory role of FASN in both cholesterol and fatty acid synthesis, a finding recently confirmed in proinflammatory macrophage activation (22). Since FASN is required for proinflammatory macrophage activation (22), our in vivo results obtained with cerulenin suggest that inhibiting proinflammatory macrophage activation delays wound healing. This is in line with the role of proinflammatory macrophages in promoting angiogenesis, an essential process during early repair (64). However, further 
studies will be required to establish the role of macrophage-specific fatty acid synthesis and the respective contribution of each pathway (cholesterol vs. fatty acid synthesis) in wound healing. This is particularly important in view of a study showing a beneficial effect of statins (simvastatin) in wound healing (65). The negative correlation between Runx2 and Fasn at the mRNA level within the module could be the result of a feedback mechanism (66), which may reflect the differences between mRNA and protein levels in terms of their correlation with each other.

In summary, we report a MMHN under Runx2 trans regulation, revealing the fatty acid/cholesterol pathways that could explain differences in physiological wound healing. Our systems genetics approach complements previous GWAS findings and highlights the BMP/Runx2 axis as a major genetic determinant of physiological wound healing. Understanding the genetic basis of wound healing will be pivotal in targeting the pathologies characterized by defective repair mechanisms.

\section{Methods}

The mouse macrophage RNA-seq data have been deposited in the NCBI's Gene Expression Omnibus database (GEO GSE112171). Phenotype data are available in Supplemental Table 1. See full methodological description of data, analyses, and experimental procedures, including Supplemental Tables 3 and 4, in the supplemental information.

\section{Author contributions}

$\mathrm{MB}, \mathrm{JHK}$, and JB carried out all experiments. AMM, NH, MI, JG, JB, and EP designed and carried out data analyses. LG contributed to the generation of RNA-seq data. JN provided genetic data. AMM, MB, $\mathrm{EP}$, and JB wrote and revised the manuscript. JB and EP designed and coordinated the study.

\section{Acknowledgments}

This work was supported by the MRC (MR/M004716/1 to JB and EP and MR/N01121X/1 to JB) and by the NMRC (grant CBRG15may062 to EP) and Duke-NUS Medical School (to EP).

Address correspondence to: Jacques Behmoaras, Centre for Inflammatory Disease, Imperial College London, Hammersmith Hospital, Du Cane Road W12 0NN, London, United Kingdom. Phone: 44.20.3313.2339; Email: jacques.behmoaras@imperial.ac.uk. Or to: Enrico Petretto, Duke-NUS Medical School, 8 College Road, 169857 Singapore, Singapore. Phone: 65.6601.5114; Email: enrico.petretto@duke-nus.edu.sg.

JN's present address is: The Francis Crick Institute, London, UK.

1. Singer AJ, Clark RA. Cutaneous wound healing. N Engl J Med. 1999;341(10):738-746.

2. Gottrup F. Optimizing wound treatment through health care structuring and professional education. Wound Repair Regen. 2004;12(2):129-133

3. Eming SA, Martin P, Tomic-Canic M. Wound repair and regeneration: mechanisms, signaling, and translation. Sci Transl Med. 2014;6(265):265sr6.

4. Murray PJ, Wynn TA. Protective and pathogenic functions of macrophage subsets. Nat Rev Immunol. 2011;11(11):723-737.

5. Eming SA, Wynn TA, Martin P. Inflammation and metabolism in tissue repair and regeneration. Science. 2017;356(6342):1026-1030.

6. Leibovich SJ, Ross R. The role of the macrophage in wound repair. A study with hydrocortisone and antimacrophage serum. Am J Pathol. 1975;78(1):71-100.

7. Lucas T, et al. Differential roles of macrophages in diverse phases of skin repair. J Immunol. 2010;184(7):3964-3977.

8. Murray PJ, Wynn TA. Obstacles and opportunities for understanding macrophage polarization. J Leukoc Biol. 2011;89(4):557-563.

9. Moreno-Moral A, Petretto E. From integrative genomics to systems genetics in the rat to link genotypes to phenotypes. Dis Model Mech. 2016;9(10):1097-1110.

10. Lusis AJ, et al. The Hybrid Mouse Diversity Panel: a resource for systems genetics analyses of metabolic and cardiovascular traits. J Lipid Res. 2016;57(6):925-942.

11. Li X, et al. Genetic control of the rate of wound healing in mice. Heredity (Edinb). 2001;86(Pt 6):668-674.

12. Cheverud JM, Lawson HA, Funk R, Zhou J, Blankenhorn EP, Heber-Katz E. Healing quantitative trait loci in a combined cross analysis using related mouse strain crosses. Heredity (Edinb). 2012;108(4):441-446.

13. Blankenhorn EP, et al. Genetic loci that regulate healing and regeneration in LG/J and SM/J mice. Mamm Genome. 2009;20(11-12):720-733.

14. Nicod J, et al. Genome-wide association of multiple complex traits in outbred mice by ultra-low-coverage sequencing. Nat Genet. 2016;48(8):912-918.

15. Flint J, Eskin E. Genome-wide association studies in mice. Nat Rev Genet. 2012;13(11):807-817. 
16. Yalcin B, et al. Commercially available outbred mice for genome-wide association studies. PLoS Genet. 2010;6(9):e1001085.

17. Yan C, et al. Epithelial to mesenchymal transition in human skin wound healing is induced by tumor necrosis factor-alpha through bone morphogenic protein-2. Am J Pathol. 2010;176(5):2247-2258.

18. Plikus MV, et al. Regeneration of fat cells from myofibroblasts during wound healing. Science. 2017;355(6326):748-752.

19. Baliga NS, et al. The state of systems genetics in 2017. Cell Syst. 2017;4(1):7-15.

20. Civelek M, Lusis AJ. Systems genetics approaches to understand complex traits. Nat Rev Genet. 2014;15(1):34-48.

21. Moreno-Moral A, Pesce F, Behmoaras J, Petretto E. Systems genetics as a tool to identify master genetic regulators in complex disease. Methods Mol Biol. 2017;1488:337-362.

22. Carroll RG, et al. An unexpected link between fatty acid synthase and cholesterol synthesis in proinflammatory macrophage activation. J Biol Chem. 2018;293(15):5509-5521.

23. Ramayo-Caldas Y, et al. Liver transcriptome profile in pigs with extreme phenotypes of intramuscular fatty acid composition. BMC Genomics. 2012;13:547.

24. Pérez-Gracia JL, et al. Selection of extreme phenotypes: the role of clinical observation in translational research. Clin Transl Oncol. 2010;12(3):174-180.

25. Heinig M, et al. A trans-acting locus regulates an anti-viral expression network and type 1 diabetes risk. Nature. 2010;467(7314):460-464.

26. Johnson MR, et al. Systems genetics identifies Sestrin 3 as a regulator of a proconvulsant gene network in human epileptic hippocampus. Nat Commun. 2015;6:6031.

27. Kang H, et al. Kcnn4 is a regulator of macrophage multinucleation in bone homeostasis and inflammatory disease. Cell Rep. 2014;8(4):1210-1224.

28. Bottolo L, et al. Bayesian detection of expression quantitative trait loci hot spots. Genetics. 2011;189(4):1449-1459.

29. Bonev B, et al. Multiscale 3D genome rewiring during mouse neural development. Cell. 2017;171(3):557-572.e24.

30. Dixon JR, et al. Topological domains in mammalian genomes identified by analysis of chromatin interactions. Nature. 2012;485(7398):376-380.

31. Symmons O, et al. Functional and topological characteristics of mammalian regulatory domains. Genome Res. 2014;24(3):390-400.

32. Lupiáñez DG, et al. Disruptions of topological chromatin domains cause pathogenic rewiring of gene-enhancer interactions. Cell. 2015;161(5):1012-1025.

33. Harmston N, Ing-Simmons E, Tan G, Perry M, Merkenschlager M, Lenhard B. Topologically associating domains are ancient features that coincide with Metazoan clusters of extreme noncoding conservation. Nat Commun. 2017;8(1):441.

34. Barutcu AR, et al. The bone-specific Runx2-P1 promoter displays conserved three-dimensional chromatin structure with the syntenic Supt3h promoter. Nucleic Acids Res. 2014;42(16):10360-10372.

35. Robertson AJ, Larroux C, Degnan BM, Coffman JA. The evolution of Runx genes II. The C-terminal Groucho recruitment motif is present in both eumetazoans and homoscleromorphs but absent in a haplosclerid demosponge. BMC Res Notes. 2009;2:59.

36. Phanstiel DH, et al. Static and dynamic DNA loops form AP-1-bound activation hubs during macrophage development. Mol Cell. 2017;67(6):1037-1048.e6.

37. Kim MS, Gernapudi R, Choi EY, Lapidus RG, Passaniti A. Characterization of CADD522, a small molecule that inhibits RUNX2-DNA binding and exhibits antitumor activity. Oncotarget. 2017;8(41):70916-70940.

38. Omura S. The antibiotic cerulenin, a novel tool for biochemistry as an inhibitor of fatty acid synthesis. Bacteriol Rev. 1976;40(3):681-697.

39. Cheverud JM, et al. Fine-mapping quantitative trait loci affecting murine external ear tissue regeneration in the LG/J by SM/J advanced intercross line. Heredity (Edinb). 2014;112(5):508-518.

40. Gallant-Behm CL, Hart DA. Genetic analysis of skin wound healing and scarring in a porcine model. Wound Repair Regen 2006;14(1):46-54

41. Valdar W, et al. Genome-wide genetic association of complex traits in heterogeneous stock mice. Nat Genet. 2006;38(8):879-887.

42. Meng W, Veluchamy A, Hébert HL, Campbell A, Colhoun HM, Palmer CNA. A genome-wide association study suggests that MAPK14 is associated with diabetic foot ulcers. Br J Dermatol. 2017;177(6):1664-1670.

43. Nakashima M, et al. A genome-wide association study identifies four susceptibility loci for keloid in the Japanese population. Nat Genet. 2010;42(9):768-771.

44. Ellinghaus E, et al. Genome-wide association analysis for chronic venous disease identifies EFEMP1 and KCNH8 as susceptibility loci. Sci Rep. 2017;7:45652.

45. Jang WG, et al. BMP2 protein regulates osteocalcin expression via Runx2-mediated Atf6 gene transcription. J Biol Chem. 2012;287(2):905-915.

46. Huang RL, Yuan Y, Tu J, Zou GM, Li Q. Opposing TNF- $\alpha$ /IL-1 $\beta$ - and BMP-2-activated MAPK signaling pathways converge on Runx2 to regulate BMP-2-induced osteoblastic differentiation. Cell Death Dis. 2014;5:e1187.

47. Okuno Y, Nakamura-Ishizu A, Kishi K, Suda T, Kubota Y. Bone marrow-derived cells serve as proangiogenic macrophages but not endothelial cells in wound healing. Blood. 2011;117(19):5264-5272.

48. Behmoaras J, Petretto E. Cell function in disease: there are more than two parties at play [published online ahead of print date] Ann Rheum Dis. https://doi.org/10.1136/annrheumdis-2018-213121.

49. Li Q, Zhao H, Xia S, Wei H, Chen F, Jin P. RUNX2 promotes epithelial differentiation of ADSCs and burn wound healing via targeting E-cadherin. Oncotarget. 2018;9(2):2646-2659.

50. Komori T. Roles of Runx2 in skeletal development. Adv Exp Med Biol. 2017;962:83-93.

51. Wu M, Chen G, Li YP. TGF- $\beta$ and BMP signaling in osteoblast, skeletal development, and bone formation, homeostasis and disease. Bone Res. 2016;4:16009.

52. Castaño-Betancourt MC, et al. Novel genetic variants for cartilage thickness and hip osteoarthritis. PLoS Genet. 2016;12(10):e1006260.

53. Zheng S, Hedl M, Abraham C. Twist1 and Twist2 contribute to cytokine downregulation following chronic NOD2 stimulation of human macrophages through the coordinated regulation of transcriptional repressors and activators. J Immunol. 
2015;195(1):217-226.

54. Chopin M, et al. RUNX2 mediates plasmacytoid dendritic cell egress from the bone marrow and controls viral immunity. Cell Rep. 2016;15(4):866-878.

55. Sawai CM, et al. Transcription factor Runx2 controls the development and migration of plasmacytoid dendritic cells. $J$ Exp Med 2013;210(11):2151-2159.

56. de Vries PS, et al. Genetic variants in the ADAMTS13 and SUPT3H genes are associated with ADAMTS13 activity. Blood. 2015;125(25):3949-3955.

57. Chauhan AK, Kisucka J, Brill A, Walsh MT, Scheiflinger F, Wagner DD. ADAMTS13: a new link between thrombosis and inflammation. J Exp Med. 2008;205(9):2065-2074.

58. Zhang Y, et al. Drug-induced regeneration in adult mice. Sci Transl Med. 2015;7(290):290ra92.

59. Vågesjö E, et al. Accelerated wound healing in mice by on-site production and delivery of CXCL12 by transformed lactic acid bacteria. Proc Natl Acad Sci USA. 2018;115(8):1895-1900.

60. Araldi E, et al. Lanosterol modulates TLR4-mediated innate immune responses in macrophages. Cell Rep. 2017;19(13):2743-2755.

61. Spann NJ, et al. Regulated accumulation of desmosterol integrates macrophage lipid metabolism and inflammatory responses. Cell. 2012;151(1):138-152.

62. Reboldi A, Dang EV, McDonald JG, Liang G, Russell DW, Cyster JG. Inflammation. 25-Hydroxycholesterol suppresses interleukin-1-driven inflammation downstream of type I interferon. Science. 2014;345(6197):679-684.

63. Wei X, et al. Fatty acid synthesis configures the plasma membrane for inflammation in diabetes. Nature. 2016;539(7628):294-298

64. Gurevich DB, et al. Live imaging of wound angiogenesis reveals macrophage orchestrated vessel sprouting and regression. EMBO J. 2018;37(13):e97786.

65. Bitto A, et al. Simvastatin enhances VEGF production and ameliorates impaired wound healing in experimental diabetes. Pharmacol Res. 2008;57(2):159-169

66. Elbaz A, Wu X, Rivas D, Gimble JM, Duque G. Inhibition of fatty acid biosynthesis prevents adipocyte lipotoxicity on human osteoblasts in vitro. J Cell Mol Med. 2010;14(4):982-991.

67. Szklarczyk D, et al. STRING v10: protein-protein interaction networks, integrated over the tree of life. Nucleic Acids Res 2015;43(Database issue):D447-D452. 